\title{
Expression of LMP-1 and Cyclin D1 protein is correlated with an unfavorable prognosis in nasal type NK/T cell lymphoma
}

\author{
WENFENG CAO ${ }^{1}$, YANXUE LIU ${ }^{1}$, HONG ZHANG $^{2}$, SHUHUA WANG ${ }^{2}$, \\ LIHUA ZHANG ${ }^{1}$, LIANYU ZHANG ${ }^{1}$ and BAOCUN SUN ${ }^{1}$ \\ ${ }^{1}$ Department of Pathology, Key Laboratory of Cancer Prevention and Therapy, and ${ }^{2}$ Department of Registry, \\ Tianjin Medical University Cancer Institute and Hospital, Tianjin 300060, P.R. China
}

Received December 17, 2007; Accepted February 19, 2008

\begin{abstract}
Nasal-type NK/T cell lymphoma (NK/TCL) is an increasingly recognized disease entity of aggressive clinical behavior. The objective of this study was to investigate the correlation between the clinical features of NK/TCL and patient prognosis. Immunohistochemical detection of EpsteinBarr virus (EBV)-associated latent membrane protein (LMP)-1, Cyclin D1 and Bcl-2 protein expression, as well as TUNEL staining, were performed. Of the 58 cases studied, $47(81 \%)$ displayed positive expression of LMP-1 protein, which was associated with the presence of B symptoms and patient prognosis. In addition, high expression of Cyclin D1 and Bcl-2 protein was observed to correlate with poor prognosis and decreased survival. More importantly, LMP-1 and Cyclin D1 appeared to be co-expressed. This, along with other parameters such as clinical stage at diagnosis and enlarged lymph nodes, could be used as an independent prognostic factor for clinical outcome, as the survival rate of NK/TCL patients was closely associated with LMP-1/Cyclin D1 co-expression, advanced clinical stage and lymph node infiltration.
\end{abstract}

\section{Introduction}

Nasal/nasopharyngeal region lymphomas encompass several distinct clinicopathologic groups redefined by the WHO classification to include NK/T cell lymphomas (NK/TCLs), unspecified peripheral $\mathrm{T}$ cell lymphomas (PTCLs) and the rare B cell lymphomas (BCLs). NK/TCL has a distinctive geographical distribution. It is most frequently observed in populations from the Far East (Korea, Japan and Singapore) (1-3) and Latin America (Mexico, Guatemala, and Peru) (4,5), whereas it is very uncommon in North America and Europe.

Correspondence to: Dr Wenfeng Cao, Department of Pathology, Key Laboratory of Cancer Prevention and Therapy, Tianjin Medical University Cancer Institute and Hospital, Tianjin 300060, P.R. China E-mail: caowenfeng@yahoo.com.cn

Key words: nasal type NK/T cell lymphoma, latent membrane protein, cyclin $\mathrm{D} 1, \mathrm{Bcl}-2$, prognosis
In addition, extranodal NK/TCLs generally occur in male adults.

Extranodal nasal-type NK/TCLs are derived from true NK cells. They initially present as a destructive midline lesion in the nasopharyngeal region, which most commonly affects the nasal cavity and other mucosal sites of the upper aerodigestive tract. Patients with this disease suffer from nasal obstruction or midfacial destruction. Although NK/TCL is usually diagnosed at an early stage, it is characterized as being very aggressive in later stages, with local destroying activity and refractoriness to treatment. Overall survival is consequently very poor (6). Almost all studies have noted that nasal-type NK/TCLs are closely related to Epstein-Barr virus (EBV) infection, as well as to abnormal expression of latent membrane protein (LMP)-1 and EB nuclear antigen (EBNA)-1 (7). To date, most research has focused on the significant expression of Cyclin D1 and Bcl-2 protein in mantle cell and follicular lymphoma, respectively. However, the correlation between the expression of these proteins in NK/TCL and patient prognosis is poorly understood.

In this study, we analyzed 58 cases of nasal-type NK/TCL using in situ hybridization of Epstein-Barr virus-encoded RNA (EBER) 1/2 and immunohistochemistry to determine immunophenotypes. We report on the correlation of LMP-1, Cyclin D1 and Bcl-2 expression with clinical outcome, and suggest potential biological markers for patients with this disease.

\section{Materials and methods}

Case selection. From the archives of the Department of Pathology, Tianjin Medical University Cancer Institute and Hospital, we selected 58 of 77 patients who had been diagnosed from January 1994 to December 2000 with nasal/ nasopharyngeal region lymphoma. All cases were histologically confirmed, with complete clinical and follow-up data. The pathologic diagnosis and classification of nasal NK/TCLs was based on previously described criteria (8): i) the histologic features of nasal and nasal-type lymphoma, ii) the expression of T cell markers (cytoplasmic CD3 and/or CD45RO), CD56 and cytotoxic molecules, iii) EBER expression by in situ hybridization, and iv) the absence of $\mathrm{B}$ cell phenotype with CD20. Cases which were negative for CD56 but positive for both cytotoxic markers and EBER were also categorized as NK/TCL. 
Clinical data collection. Clinical information was obtained from hospital medical records. Survival status of all of the patients was available from the Department of Registry, Tianjin Medical University Cancer Hospital. Of the 58 cases of nasaltype NK/TCL selected, 18 were in the nasopharynx, 11 in the tonsils, 8 in the pharynx, 6 in the nasal cavity, 5 in the soft palate, 4 in the tongue, 4 in the paranasal sinuses and 2 in the epiglottis. The following clinical data were also collected: patient sex ratio, age distribution, Ann Arbor stage, the presence of B symptoms, enlarged lymph nodes in other regions, type of treatment (surgery, chemotherapy or radiotherapy), response to treatment and survival status.

Histologic analysis. The morphologic features of the cases were evaluated using tissue sections which had been formalinfixed, paraffin-embedded and H\&E-stained independently by WF.C. and LY.Z. Tissues were examined in order to distinguish benign from malignant tumors, to initially determine the pathologic type, and to assess the presence of necrosis and angioinvasion.

Immunohistochemistry. For immunohistochemical analysis, consecutive $4-\mu \mathrm{m}$ sections were mounted on adhesive-coated slides and stained with monoclonal antibody anti-CD45RO (UCH-L1, Santa Cruz), and antibodies anti-CD3 (PS1, Santa Cruz), anti-CD20 (L26, Santa Cruz), anti-CD56 (123C3, Beijing Zhongshan), anti-TIA-1 (TIA-1, Santa Cruz) and anti-LMP-1 (CS1-4, Dakopatts), followed by streptavidinbiotin. Antigens were retrieved by microwave pre-treatment in citrate buffer ( $\mathrm{pH}$ 6.0) for $4 \mathrm{~min}$ at high power followed by 10 min maintenance at low power. For the negative controls, PBS was substituted for the primary antibody.

Additional staining was performed with mono/polyclonal primary antibodies against Bcl-2 (100, Santa Cruz) and Cyclin D1 (SP4, Maixin-Bio) using the aforementioned method. To evaluate the protein immunostaining, the percentage of positively-stained cancer cells was calculated using a semi-quantitative method, and defined as follows: 0, absence or $<10 \%$ staining of tumor cells; $1+$, weak and/or $10-25 \%$ staining; $2+$, moderate and/or 26-50\% staining; $3+$, strong and/or $>50 \%$ staining of tumor cells.

In situ hybridization. In situ hybridization (ISH) for EBER was performed using the EBER Detection Kit (Triplex International Biosciences, Fuzhou) and conducted according to the manufacturer's instructions. Briefly, paraffin-embedded sections were deparaffinized with xylene, hydrated with ethanol, pre-digested with proteinase $\mathrm{K}$ and quenched by endogenous peroxidase activity. Next, $20 \mu 1$ of the probe hybridization solution was applied. Sections were coverslipped and incubated for $90 \mathrm{~min}$ at $55^{\circ} \mathrm{C}$, then at $37^{\circ} \mathrm{C}$ overnight. Subsequently, the covers were allowed to drain and were washed in PBS at $48^{\circ} \mathrm{C}$ for $3 \times 5 \mathrm{~min}$. For detection, $50 \mu \mathrm{l}$ of blocking solution was applied for $5 \mathrm{~min}$, and the sections were incubated with HRP conjugated to biotin-labeled antibody to indirectly combine digoxigenin-labeled oligonucleotide probes for $30 \mathrm{~min}$, then washed in PBS followed by DAB buffer for $\sim 10 \mathrm{~min}$. Finally, the slides were washed and counterstained with Mayer's haematoxylin. The control procedures, including positive and negative control sections, were conducted simultaneously.
Table I. Association of LMP-1 expression with clinical characteristics and selected biological markers of NK/TCLs.

\begin{tabular}{|c|c|c|c|c|}
\hline \multirow[b]{2}{*}{ Variables } & \multirow[b]{2}{*}{ No. } & \multicolumn{2}{|c|}{ LMP-1 expression } & \multirow[b]{2}{*}{$\mathrm{P}$-value } \\
\hline & & Negative & Positive & \\
\hline \multicolumn{5}{|l|}{ Stage at diagnosis } \\
\hline I & 15 & $2(13.3)$ & $13(86.7)$ & 0.539 \\
\hline II & 31 & $7(22.6)$ & $24(77.4)$ & \\
\hline III & 7 & $2(28.6)$ & $5(71.4)$ & \\
\hline IV & 5 & $\begin{array}{ll}0 & (0.0)\end{array}$ & $5(100.0)$ & \\
\hline \multicolumn{5}{|l|}{ B symptoms } \\
\hline Absence & 41 & $11(26.8)$ & $30(73.2)$ & 0.024 \\
\hline Presence & 17 & $\begin{array}{ll}0 & (0.0)\end{array}$ & $17(100.0)$ & \\
\hline \multicolumn{5}{|l|}{ Enlarged lymph nodes } \\
\hline Absence & 17 & $2(11.8)$ & $15(88.2)$ & 0.368 \\
\hline Presence & 41 & $9(22.0)$ & $32(78.0)$ & \\
\hline \multicolumn{5}{|l|}{ Treatment } \\
\hline Chemotherapy alone & 14 & $2(14.3)$ & $12(85.7)$ & 0.436 \\
\hline Chemo-radiotherapy & 34 & $9(26.5)$ & $25(73.5)$ & \\
\hline Radiotherapy alone & 4 & $0 \quad(0.0)$ & $4(100.0)$ & \\
\hline Surgery alone & 3 & $0 \quad(0.0)$ & $3(100.0)$ & \\
\hline ASCT & 3 & $\begin{array}{lll}0 & (0.0)\end{array}$ & $3(100.0)$ & \\
\hline \multicolumn{5}{|l|}{ Response to treatment } \\
\hline $\mathrm{CR}$ & 15 & $5(33.3)$ & $10(66.7)$ & 0.151 \\
\hline PR & 36 & $6(16.7)$ & $30(83.3)$ & \\
\hline NR & 7 & $\begin{array}{lll}0 & (0.0)\end{array}$ & $7(100.0)$ & \\
\hline \multicolumn{5}{|l|}{ Prognosis } \\
\hline Living & 30 & $9(30.0)$ & $21(70.0)$ & 0.043 \\
\hline Deceased & 28 & $2(7.1)$ & $26(92.9)$ & \\
\hline \multicolumn{5}{|l|}{ Cyclin D1 expression } \\
\hline Negative & 17 & $7(63.6)$ & $10(21.3)$ & 0.010 \\
\hline Positive & 41 & $4(36.4)$ & $37(78.7)$ & \\
\hline \multicolumn{5}{|l|}{ Bcl-2 expression } \\
\hline Negative & 24 & $6(54.5)$ & $18(38.3)$ & 0.325 \\
\hline Positive & 34 & $5(45.5)$ & $29(61.7)$ & \\
\hline \multicolumn{5}{|l|}{ TUNEL staining (AI) } \\
\hline Negative & 19 & $4(36.4)$ & $15(31.9)$ & 0.777 \\
\hline Positive & 39 & $7(63.6)$ & $32(68.1)$ & \\
\hline
\end{tabular}

ASCT, autologous stem cell transplantation; CR, complete remission; PR, partial remission; NR, no response.

TUNEL assay. Tissue cell apoptosis was detected using a commercially-available TUNEL [terminal deoxynucleotidyl transferase (TdT)-mediated deoxy uridine triphosphate nick end labeling] assay (TdT Fragel DNA Fragmentation In situ Apoptosis Detection Kit, Beijing Zhongshan) as per the manufacturer's instructions. Briefly, formalin-fixed, paraffinembedded sections were deparaffinized and hydrated using graded alcohols, treated with proteinase $\mathrm{K}$ and $0.3 \% \mathrm{H}_{2} \mathrm{O}_{2}$, then incubated with a labeling reaction mixture consisting of TdT and fluorescein dUTP in a humid box for $1 \mathrm{~h}$ at $37^{\circ} \mathrm{C}$. Slices were then combined with POD-horseradish peroxidase, 

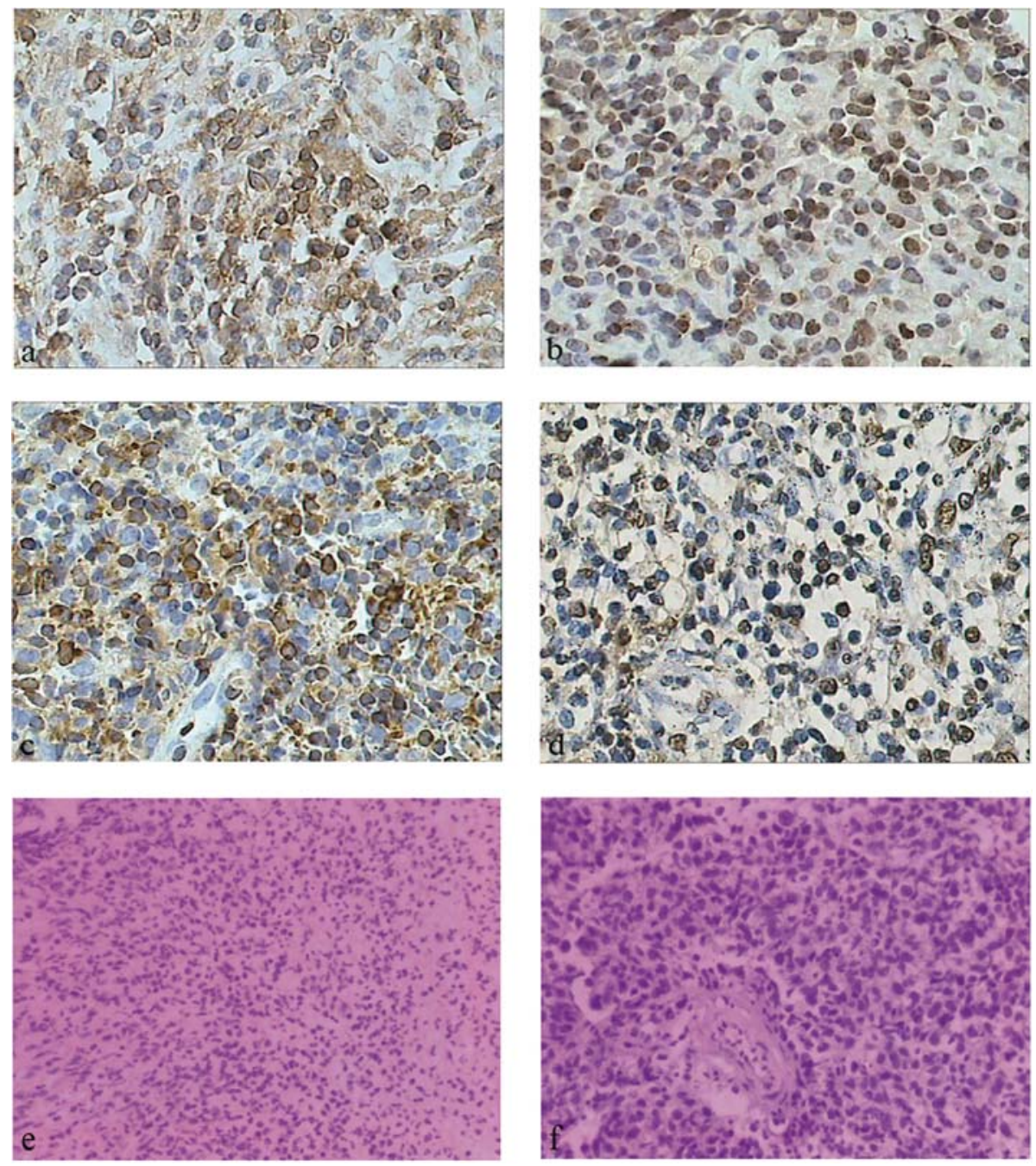

Figure 1. IHC staining of NK/T cell lymphoma tissues. (a) LMP-1 protein positively expressed in the cytoplasm of NK/TCL cells. (b) Positive expression of Cyclin D1 protein in the nuclei of lymphoma cells. (c) Bcl-2-positive particles in the cytoplasm and cell plasma membrane of lymphoma cells. (d) Apoptosis staining in the nucleus of NK/TCL cells (original magnification x200). (e) NK/TCL with small-sized cells (original magnification x100). (f) NK/TCL with medium-sized cells exhibiting slightly irregular nuclei and infiltrating a blood vessel (original magnification x200).

stained with DAB and counter-stained with Mayer's haematoxylin. TUNEL-positive cells exhibited dark brown staining of the nucleus, suggesting the internucleosomal cleavage of DNA. All other cells appeared blue due to the counterstain. Controls were treated in the same fashion, except that fluorescein dUTP was not included in the labeling reaction. The apoptotic index (AI) was calculated as follows: $\mathrm{AI}=$ number of apoptotic cells/ total number of cells $\mathrm{x} 100 \%$ The staining of $>10 \%$ cells was regarded as positive.

Statistical analysis. Statistical analysis was performed using SPSS software package 11.0 (SPSS, Chicago, IL). Correlations between LMP-1 expression and clinicopathologic variables were analyzed using Pearson's $\chi^{2}$ test. The same method was used to test for associations between LMP-1 and proliferative and apoptotic indices such as Cyclin D1, PCNA, Bcl-2 expression and apoptosis staining. The Kaplan-Meier method was used to analyze survival, and univariate and multivariate analyses in a Cox proportional hazards model to evaluate the impact of clinicopathologic variables and proliferative and apoptotic indices on survival. All statistical tests were 2-sided, and P-values $\leq 0.05$ were considered statistically significant.

\section{Results}

Clinical features. The main clinicopathologic characteristics of patients with nasal-type NK/TCL are listed in Table I. The 77 cases of nasal/nasopharyngeal region lymphomas were categorized into three immunophenotypic groups: i) nasal-type NK/TCLs (58 cases; Fig. 1e and f), with a morphology ranging from small cells (13 cases) or medium-sized cells (24 cases) to large transformed cells ( 21 cases), in which necrosis was observed in $66 \%$ of the cases and angioinvasion in $45 \%$. All NK/TCLs showed positive staining for $\mathrm{CCD} 3$, TIA-1, CD45RO and EBER-1 and negative staining for CD20, while CD56 expression was present in $28 \%$ of the cases. ii) PTCLs (12 cases), in which CD56, TIA-1 and EBER-1 were negative, but CD45RO and CD3 exhibited positivity. iii) BCLs (7 cases), in which all cancer cells demonstrated marked CD20 positivity only.

In the NK/TCL subgroup, there was a preponderance of male to female patients with a ratio of $1.52: 1$. The median age at diagnosis was 45.5 years (range 10-78 years). Nasal obstruction was the most common initial symptom. According to the Ann Arbor stage of lymphoma, at the time of diagnosis 

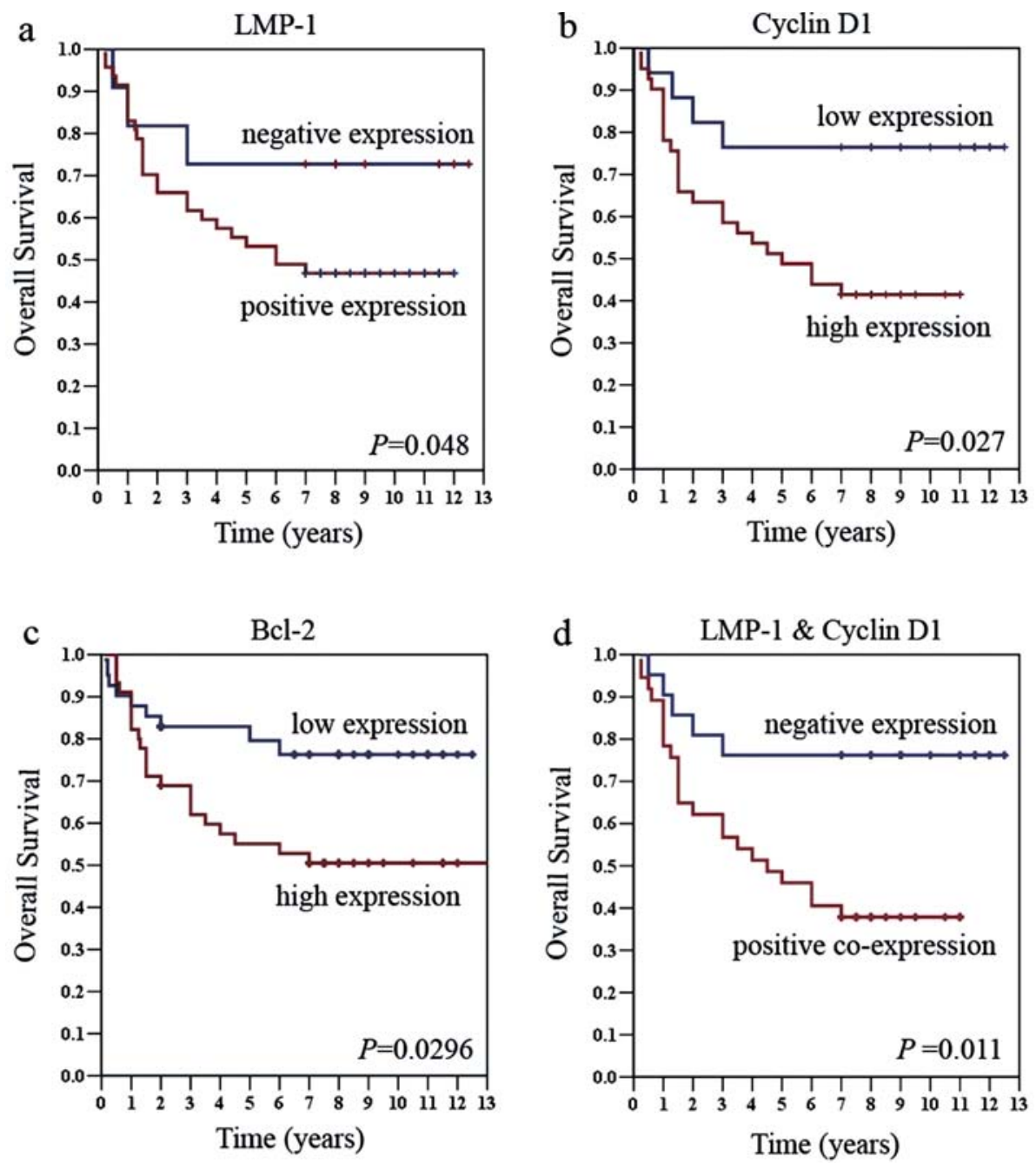

Figure 2. Correlation between (a) LMP-1 protein expression, (b) Cyclin D1 protein expression, (c) Bcl-2 protein, and (d) LMP-1/Cyclin D1 protein co-expression with overall patient survival. Kaplan-Meier survival analysis revealed that patients with LMP-1, Cyclin D1 and Bcl-2 expression, and in particular LMP-1/ Cyclin D1 co-expression, had significantly shorter survival.

15 patients were in stage I, 31 in stage II and 12 in advanced stages (7 in stage III and 5 in stage IV) of the disease. Nine patients progressed from the early stage (I-II) to advanced stage (III-IV). Other clinical features, such as B symptoms, were encountered in 17 patients (29.3\%), and enlarged lymph nodes were present in 41 patients $(70.7 \%)$.

Of the $58 \mathrm{NK} / \mathrm{TCL}$ patients, some received just chemotherapy (14 cases), others a combination of chemo and radiotherapy (34 cases), and some radiotherapy alone (4 cases). Three patients underwent autologous stem cell transplantation (ASCT) combined with chemo and radiotherapy, and 3 underwent surgery alone. Fifteen patients experienced complete remission (CR) (25.9\%) and 36 partial remission (PR) (62.1\%), while 7 patients were refractory to treatment (12\%).

LMP-1 protein expression in nasal-type NK/TCL. LMP-1 protein was expressed mainly in the cytoplasm (Fig. 1a). Positive staining was noted in 47 cases of NK/TCL (81\%), and statistical analysis indicated a significant relationship between LMP-1 protein expression and clinical characteristics, including the presence of $\mathrm{B}$ symptoms $(\mathrm{P}=0.024)$ and patient prognosis $(\mathrm{P}=0.043)$. However, no significant association was found between LMP-1 expression and other clinical parameters, such as Ann Arbor stage, enlarged lymph nodes in other regions, type of treatment or response to treatment (Table I).

Protein expression and apoptotic indices in nasal-type NK/ TCL. Cyclin D1 protein expression was localized to the nuclei of lymphoma cells (Fig. 1b). Of the $58 \mathrm{NK} / \mathrm{TCL}$ cases, 41 were positive $(70.7 \%)$ with an immune reactive score (mean \pm SD) of 31.04 \pm 13.796 . Importantly, moderate-to-strong staining of Cyclin D1 was associated with poor prognosis $(\mathrm{P}=0.015)$.

Bcl-2 expression was localized to the cytoplasm and cell plasma membrane (Fig. 1c) and was positive in 34 cases $(58.6 \%)$. The immune reactive score (mean \pm SD) was $39.16 \pm$ 18.595, indicating that the high-level expression of Bcl-2 protein is linked to mortality in this type of lymphoma patient $(\mathrm{P}=0.014)$.

Cell apoptosis staining (TUNEL) was present in the nuclei of cells (Fig. 1d). Positive staining was observed in 39 cases $(67.2 \%)$ of NK/TCL, with an immune reactive score of $42.02 \pm$ 22.900. However, no significant association was observed between cell apoptosis expression and patient prognosis $(\mathrm{P}=0.553, \mathrm{P}=0.113)$.

Additionally, statistical analysis showed a strong correlation between LMP-1 and Cyclin D1 protein expression $(\mathrm{P}=0.010)$, 
Table II. Univariate and multivariate Cox survival analysis.

\begin{tabular}{lc}
\hline Variable & P-value \\
\hline Univariate & \\
Age & 0.510 \\
Stage at diagnosis & 0.070 \\
Enlarged lymph nodes & 0.050 \\
Treatment & 0.210 \\
B symptoms & 0.532 \\
Response to treatment & 0.604 \\
LMP-1 expression & 0.915 \\
Cyclin D1 expression & 0.874 \\
LMP-1/Cyclin D1 co-expression & 0.271 \\
Bcl-2 expression & 0.633 \\
Apoptosis staining & 0.292 \\
Multivariate & \\
Stage at diagnosis & 0.013 \\
Enlarged lymph nodes & 0.050 \\
LMP-1/Cyclin D1 co-expression & 0.026 \\
\hline
\end{tabular}

but no significant association was found between LMP-1 and Bcl-2 expression or apoptotic markers (Table I).

Patient survival. Kaplan-Meier analysis of these 58 nasal NK/TCL cases, with a mean follow-up of 84 months, showed a significantly lower survival rate in patients with LMP-1, Cyclin D1 and Bcl-2 expression than in those without. Namely, LMP-1, Cyclin D1, and Bcl-2 protein expression were all associated with decreased overall survival $(\mathrm{P}=0.048, \mathrm{P}=0.027$, $\mathrm{P}=0.0296)$. In particular, the co-expression of LMP-1 and Cyclin D1 proteins was associated with an obvious decrease in patient survival $(\mathrm{P}=0.011)$ (Fig. 2a-d).

Univariate survival analysis, using the Cox proportional hazards model, showed that only the variable of enlarged lymph nodes was significantly related to low survival. In multivariate survival analysis, three factors - clinical stage at diagnosis, enlarged lymph nodes and LMP-1 and Cyclin D1 protein co-expression - were independent prognostic values for an aggressive course in nasal NK/TCLs (Table II).

\section{Discussion}

Nasal-type NK/TCL is a distinctive type of extranodal lymphoma with a unique immunophenotype of NK cell derivation $(9,10)$. It is also highly aggressive and is closely associated with EBV, thus expressing EBNA-1 and LMP-1 (11). Its histopathology is characterized by an angiocentric/angiodestructive growth pattern, with fibrinoid changes in the blood vessels. Coagulative necrosis very often occurs as a consequence of vascular occlusion. The cytological spectrum of NK/TCL is very broad, but most cases are composed of medium-to-large sized transformed cells with irregular nuclei and granular chromatin. Mitotic figures are also commonly found.
Certain groups of viruses are closely associated with lymphomagenesis in humans. EBV, classified as a lymphocrypto $\gamma$ herpes virus, is an etiological agent of lymphoproliferative disorders including Burkitt's lymphoma and infectious mononucleosis in humans $(12,13)$. Recent studies on malignant lymphoma have revealed that EBV contributes to the development of Hodgkin's and non-Hodgkin's lymphoma, including T cell lymphoma such as NK/TCL $(14,15)$. In addition, in the latent state of the EBV product, the viral oncogene protein LMP-1 may participate in the pathogenesis of NK/TCLs. In this study, we have confirmed the presence of EBV in lymphoma tissues, and have explored the role of the LMP-1 protein in NK/TCLs.

Out of 77 cases of nasal/nasopharyngeal region lymphomas, 58 were cases of NK/TCL, 12 of PTCL and 7 of BCL. Of these, we chose to study LMP-1 protein expression status and its correlation with certain clinical tumor characteristics in the NK/TCLs group. Among the NK/TCL cases, 47 out of $58(81 \%)$ had LMP-1 expression. Association analysis of LMP-1 expression with clinical parameters indicated that it was obviously related to the presence of B symptoms and patient survival status. In other words, expression of LMP-1 protein indicated a poor prognosis, including the appearance of B symptoms and patient death due to the rapid progression of the disease. However, other clinical characteristics, such as clinical stage, enlarged lymph nodes, type of treatment and response to treatment, did not seem to be correlated with LMP-1 protein expression.

Nasal-type NK/TCL is a kind of nasal/nasopharyngeal region lymphoma, a heterogeneous group of non-Hodgkin's lymphomas with a wide spectrum of clinicopathologic features. Cell proliferation mechanisms may play a role in lymphomagenesis and progression, and diverse mechanisms resulting in the deregulation of apoptotic pathways are involved in human lymphomagenesis (16). As a result, the evaluation of the apoptotic rate of NK/TCLs may contribute to our understanding of the underlying biological mechanisms of NK/T cell tumorigenesis and may also have prognostic implications. In this study, we assessed 58 cases of NK/TCL for the expression of proliferative and apoptotic indices, including Cyclin D1, Bcl-2 and the apoptosis index, and examined their relationship to prognosis and survival.

Cyclin D1 is, in brief, an important regulator of cell cycle progression and can function as a transcriptional co-regulator. The overexpression of Cyclin D1 has been linked to the development and progression of cancer through the promotion of cell proliferation. The Bcl-2 family of proteins are among the most crucial regulators of apoptosis, and perform a vital function regarding the decision of whether a cell will live or die. In addition, several studies have now revealed that members of the Bcl-2 family can interface with the cell cycle, DNA repair/recombination and cellular senescence.

Our present results demonstrate that NK/TCL is a tumor with a high proliferative and apoptotic activated state. In detail, out of the $58 \mathrm{NK} / \mathrm{TCL}$ cases $41(70.7 \%)$ exhibited Cyclin D1 staining, $34 \mathrm{Bcl}-2$ expression (58.6\%) and 39 cell apoptosis $(67.2 \%)$. Immune reactive scores were $31.04 \pm 13.796,39.16 \pm$ 18.595 and $42.02 \pm 22.900$, respectively. In addition, it is important to note that significant associations were found between high-level expression of Cyclin D1 or Bcl-2 protein and patient 
prognosis and survival. However, no similar association was found between apoptosis staining and patient prognosis. Of interest, we found a strong association between LMP-1 and Cyclin D1 expression, as opposed to other proliferative and apoptotic proteins. Our findings differ from those of other studies, which have reported that in primary brain lymphomas the high expression of LMP-1 protein is correlated with the expression of $\mathrm{Bcl}-2$ oncoprotein, and suggest a transactivation of Bcl-2 by LMP-1 as reported in vitro (17).

Multivariate survival analysis in this series of NK/TCL showed that clinical stage at diagnosis, enlarged lymph nodes and LMP-1/Cyclin D1 protein co-expression independently predict overall survival. Meanwhile, there exists an obvious correlation between LMP-1 and Cyclin D1 protein expression. These can independently decrease NK/TCL patient overall survival; together, they promote the progression of NK/TCLs. Based on our results, LMP-1/Cyclin D1 co-expression patterns appear to be of importance, and are significantly associated with the decreased overall survival of patients with NK/TCLs.

Collectively, the data suggest that LMP-1 protein expression is correlated to certain NK/TCL clinical parameters, such as the presence of $\mathrm{B}$ symptoms and patient prognosis. The high-level expression of proliferative factor Cyclin D1 and apoptotic factor Bcl-2 is also linked to poor prognosis and decreased overall survival. In addition, a significant positive association was found between the expression of LMP-1 and Cyclin D1 protein. Their co-expression, along with clinical stage at time of diagnosis and enlarged lymph nodes, can act as independent prognostic factors for the prediction of clinical outcome. In summary, our results indicate that the expression of EBV-associated LMP-1 protein and the production of Cyclin D1 and Bcl-2 may favor the progession of NK/TCL. LMP-1/Cyclin D1 co-expression, advanced clinical stage and lymph node infiltration of other regions are also associated with significantly decreased patient survival.

\section{Acknowledgements}

This work was supported by Tianjin Medical University and Cancer Hospital Research Funds.

\section{References}

1. Lee J, Kim WS, Park YH, et al: Nasal-type NK/T cell lymphoma: clinical features and treatment outcome. Br J Cancer 92: 1226-1230, 2005

2. Ohsawa M, Nakatsuka S, Kanno H, et al: Immunophenotypic and genotypic characterization of nasal lymphoma with polymorphic reticulosis morphology. Int J Cancer 81: 865-870, 1999.

3. Ng SB, Lai KW, Murugava S, et al: Nasal-type extranodal natural killer/T-cell lymphomas: a clinicopathologic and genotypic study of 42 cases in Singapore. Mod Pathol 17: 1097-1107, 2004.

4. Van de Rijn M, Bhargava V, Molina-Kirsch H, et al: Extranodal head and neck lymphomas in Guatemala: high frequency of Epstein-Barr virus-associated sinonasal lymphomas. Hum Pathol 28: 834-839, 1997.

5. Arber DA, Weiss LM, Albujar PF, et al: Nasal lymphomas in Peru. High incidence of T-cell immunophenotype and EpsteinBarr virus infection. Am J Surg Pathol 17: 392-399, 1993.

6. Pagano L, Gallamini A, Trape G, et al: NK/T-cell lymphomas 'nasal type': an Italian multicentric retrospective survey. Ann Oncol 17: 794-800, 2006

7. Takahara M, Kishibe K, Bandoh N, et al: P53, N- and K-Ras, and beta-catenin gene mutations and prognostic factors in nasal NK/T-cell lymphoma from Hokkaido, Japan. Hum Pathol 35: 86-95, 2004.

8. Jaffe ES, Krenacs L, Kumar S, et al: Extranodal peripheral T-cell and NK-cell neoplasms. Am J Clin Pathol 111: S46-S55, 1999.

9. Jaffe ES: Classification of natural killer (NK) cell and NK-like T-cell malignancies. Blood 87: 1207-1210, 1996.

10. Kinney MC: The role of morphologic features, phenotype, genotype, and anatomic site in defining extranodal T-cell or NK-cell neoplasms. Am J Clin Pathol 111: S104-S118, 1999.

11. Takahara M, Kis LL, Nagy N, et al: Concomitant increase of LMP1 and CD25 (IL-2-receptor alpha) expression induced by IL-10 in the EBV-positive NK lines SNK6 and KAI3. Int J Cancer 119: 2775-2783, 2006.

12. Straus SE, Cohen JI, Tosato G, et al: NIH conference. EpsteinBarr virus infections: biology, pathogenesis, and management. Ann Intern Med 118: 45-58, 1993.

13. Zur Hausen H, Schulte-Holthausen H, Klein G, et al: EBV DNA in biopsies of Burkitt tumours and anaplastic carcinomas of the nasopharynx. Nature 228: 1056-1058, 1970.

14. Flavell KJ and Murray PG: Hodgkin's disease and the EpsteinBarr virus. Mol Pathol 53: 262-269, 2000.

15. Gaal K, Sun NC, Hernandez AM, et al: Sinonasal NK/T-cell lymphomas in the United States. Am J Surg Pathol 24: 1511-1517, 2000.

16. Rassidakis GZ, Jones D, Thomaides A, et al: Apoptotic rate in peripheral T-cell lymphomas. A study using a tissue microarray with validation on full tissue sections. Am J Clin Pathol 118: 328-334, 2002.

17. Raphael M: Role of Epstein-Barr virus in lymphoproliferative disorders. Bull Acad Natl Med 181: 999-1007, 1997. 